\title{
Behavior of Rubber Materials under Exposure to High Electric Fields
}

\author{
A. Candela, J. Holboell, M. Henriksen \\ Technical University of Denmark, Department of Electrical Engineering \\ Kgs. Lyngby, Denmark
}

\begin{abstract}
The effect of high electrical stress on rubber materials is investigated by performing breakdown tests and tracking resistance tests on selected samples. The study is focused on the relationship between the dielectric strength and the thickness of the samples, as well as the influence of the interfaces between different layers of material. Tracking resistance tests are also performed on the rubber material. The purpose is to provide a complete study of the applicability of the rubber material in thunderstorm environments.
\end{abstract}

\section{Introduction}

In the recent years, new technologies have been developed to increase the efficiency of wind turbine blades, some of which involve the use of rubber materials in the blade structure. Amongst these technologies, the deformable flaps aim at reducing the load on the blade, thus alleviating the fatigue strain on the whole wind turbine [1]. This is achieved by installing a rubber flap in the trailing edge of the blade (Fig. 1), and controlling its deflection using a compressed-air system. Once installed in the blade, the rubber flap will be subjected to severe ambient conditions. This paper is focused on the effect of high electric fields on the electrical performance of rubber materials.

During their lifetime wind turbines are repeatedly exposed to high electric fields from thunderstorms, which degrade progressively the insulating properties of the blade materials [2]. The interaction between the thunderstorm electric field and the fiberglass material usually used in wind turbines has been widely studied, [3]. However, the behavior of the rubber material in a thunderstorm environment is not fully known and therefore needs to be assessed.

The IEC standard on lightning protection of wind turbines [2] defines the tests to be performed in wind turbines to reproduce the effects of direct lightning strike. However, there is a need for tests aimed at assessing the effects of repeated high electric field and discharge exposure on the insulating materials of the blade. In this study, the rubber behavior under high electric fields is investigated by performing breakdown and tracking resistance tests. These methods have been used previously to evaluate the performance of blade insulating materials against lightning [3], [4]. The criterion used to evaluate the tests results on fiberglass was to affect the material as it was seen in blades in service.
Samples of four different rubber materials have been subjected to breakdown and tracking tests. The samples and setups used in the tests are described in section 2 . Section 3 summarizes the test results, comparing the different rubber materials. The relationship between the breakdown strength and the thickness of the samples and the influence of the interfaces between the layers of material are also investigated and described in this section. Finally, the outcome of the tests is discussed in section 5 , where the suitability of rubber materials in wind turbine blades is discussed.

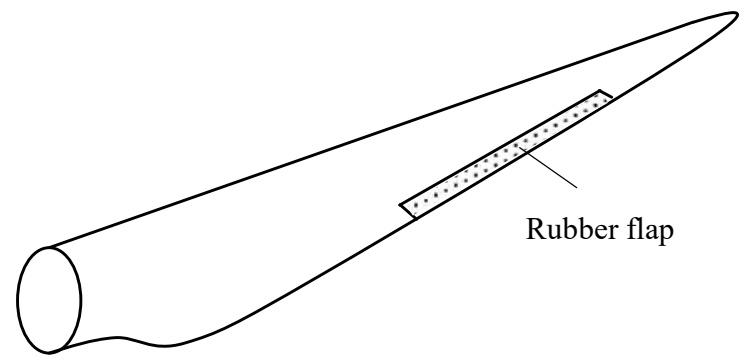

Fig.1 - Wind turbine blade equipped with a rubber flap system, installed on the blade trailing edge.

\section{Materials and tests description}

Four different rubber materials are used for both the breakdown and the tracking resistance tests. These materials are a representative selection of different types of rubber:

- Santoprene 121-73W175 (Polyolefin elastomer)

- $\quad$ Silicone rubber 5060-5

- $\quad$ PUR 8070-3 (Polyurethane)

- EPDM 2165-1 (Ethylene Propylene Diene monomer (M-class))

\subsection{Breakdown strength tests}

The specimens used for this test are square shaped, with a side length of $100 \mathrm{~mm}$. Each material has been tested with a thickness of 1, 2, 3 and $4 \mathrm{~mm}$.

The sample is placed between two electrodes inside a container filled with silicone oil (Fig. 2). The upper electrode is spherical, with a diameter of $12.5 \mathrm{~mm}$, and it is connected to the high impulse voltage generator. The lower electrode is cylindrical with rounded edges, with a diameter of $70 \mathrm{~mm}$, and is connected to ground. The purpose of the silicone oil is to increase the electrical breakdown of the media around the specimen, in order to prevent side flashovers.

The test follows the procedure described in [5]. It consists of applying a high voltage impulse with a rise 
time and decay to half value of 1.2 and $50 \mu \mathrm{s}$ respectively, according to [6]. The test starts at relatively low voltage, where there is no risk of breakdown, and it is increased progressively until the breakdown of the material is reached.

This procedure is repeated 6 times. Each time, the peak value of the voltage impulse that produces breakdown and the peak value of the withstand voltage previous to breakdown are measured. The breakdown and withstand voltages of each material found in section 3 correspond to the average value of the measurements.

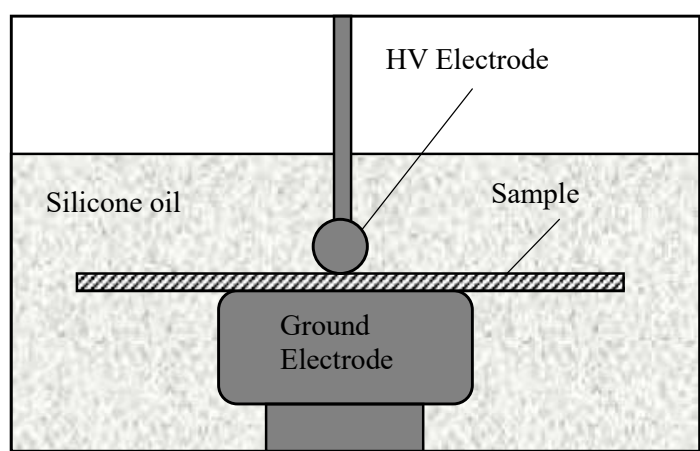

Fig.2 - Breakdown test setup: rubber sample placed between the high voltage and ground electrodes, inside a container filled with silicone oil.

\subsection{Tracking resistance tests}

The setup arrangement and the test procedure follow the standard setup described in [7]. The specimens are mounted on an insulating support, which stands at an angle of 45 degrees from the horizontal. Two electrodes are placed on the top and the bottom of the sample, connected to high voltage and ground respectively (Fig. $3)$. The specimens are arranged in sets of 5 samples. Each sample is $50 \times 120 \mathrm{~mm}$, with a thickness of $2 \mathrm{~mm}$ (Fig. 4).

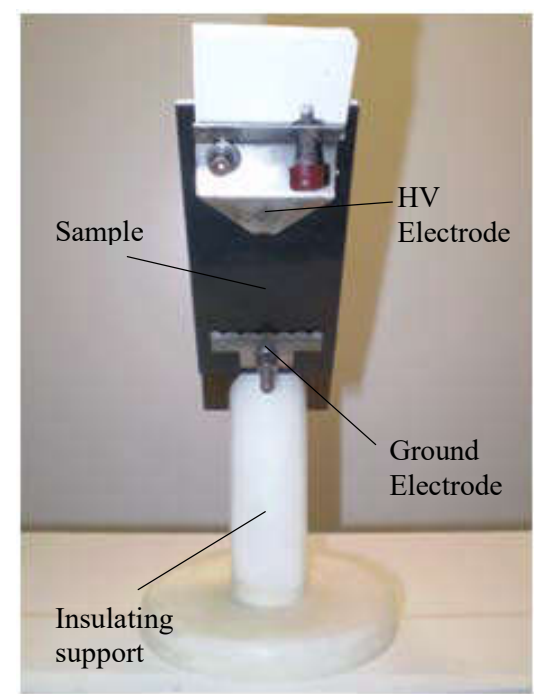

Fig.3 - Tracking resistance test setup. Sample of rubber material mounted on the insulating support with the electrodes.

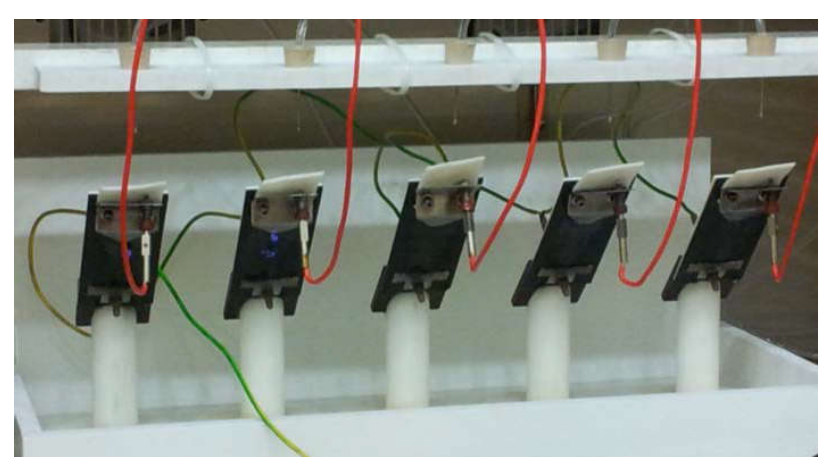

Fig.4 - Tracking resistance test setup. Set of 5 samples mounted on the supports.

The procedure follows the Method 2, according to [7]: stepwise tracking voltage. It mainly consists of applying a sequence of $\mathrm{AC}$ voltage levels across the sample while a contaminant solution based on $\mathrm{NH}_{4} \mathrm{Cl}$ is flowing over the sample lower surface. The initial voltage is chosen in such a way that no sample will fail during the three first steps, and is increased by $250 \mathrm{~V}$ every hour. The end-point criterion used in this test is "End-point criterion A: the value of current through the specimen exceeds $60 \mathrm{~mA}$ ". In order to determine when the current is over the maximum value allowed, a fuse is installed in the HV circuit of each sample.

\section{Tests results}

This section summarizes the results of the breakdown strength and the tracking resistance tests on the rubber materials.

\subsection{Breakdown strength tests}

The breakdown strength tests were performed on 6 samples of each thickness, for the four different rubber materials. The average breakdown and withstand voltages for each material and thickness are displayed in Fig 5.

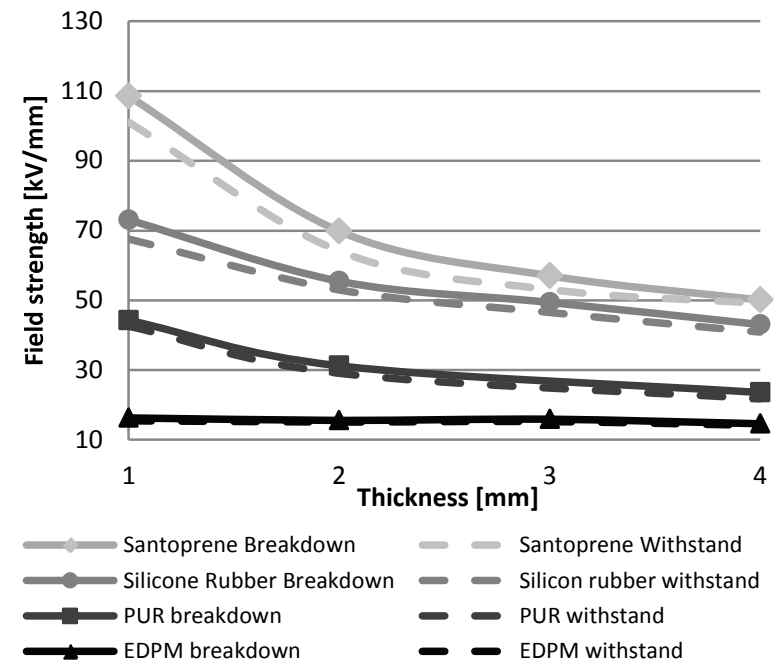

Fig.5 - Breakdown and withstand field strength of the tested materials, for thicknesses from 1 to $4 \mathrm{~mm}$. 
According to the test results, the Santoprene material presents the highest breakdown field strength of 110 $\mathrm{kV} / \mathrm{mm}$, followed by silicone rubber showing 72 $\mathrm{kV} / \mathrm{mm}$. It is also observed that the breakdown strength decreases when increasing the thickness of the sample. This can be explained by the so-called volume effect, where an increase of the material thickness involves a higher probability of impurities or microscopic defects. These inhomogeneities, normally small particles and air bubbles, enhance the electric field around or inside them, and lead to an earlier breakdown of the material. The volume effect can be found in all the tested materials except the EDPM. Considering that this material shows a very low breakdown strength, the negative influence of possible impurities is less dominant.

In order to study further the volume effect and the influence of interfaces, additional tests were performed to Santoprene, the material showing the highest breakdown strength. These tests consisted of testing two layers of material together. The results were compared to the breakdown strength of a single layer with the same total thickness and with the theoretical calculation of the breakdown strength obtained from the independent layers.

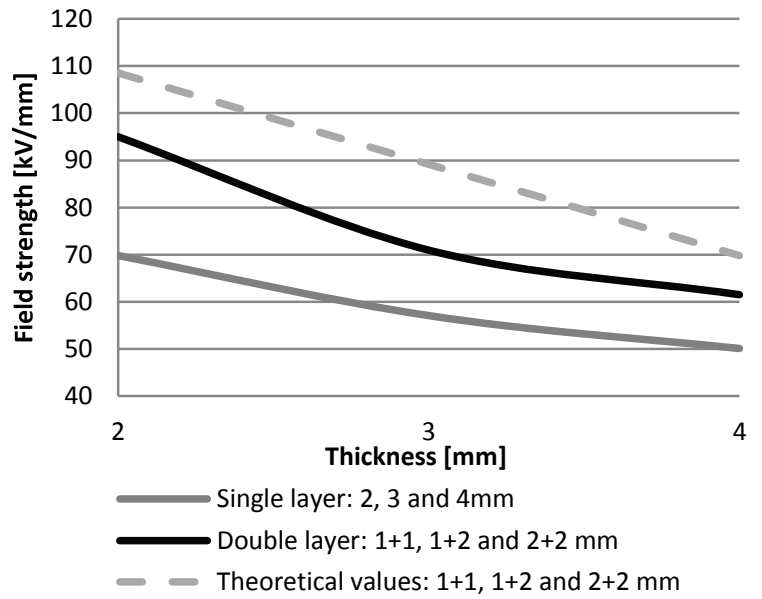

Fig.6 - Comparison of breakdown field strength between single-layer setup, double-layer setup and theoretical values for the double layer setup calculated from the breakdown strength of the independent layers.

The test reveals that the double-layer setup has higher breakdown strength than the single-layer setup. At a first glance, this may be surprising, since we have an additional interface in the material. On the other hand each layer of the double-layer setup presents fewer impurities per volume than the thicker single-layer, which makes the result more plausible. It is also found that the breakdown strength of the double-layer setup is lower than the value calculated from each independent layer. This fact indicates that in the double-layer setup, the breakdown occurs first in the weakest layer, directly followed by the other layer. Therefore, the weakest layer determines the breakdown of both layers. This phenomenon is more evident in the case of mixed thickness $(1+2 \mathrm{~mm})$, where the difference between the calculated and the actual breakdown strength of the double-layer is greater.

\subsection{Tracking resistance tests}

The tracking resistance tests were performed on sets of five samples of the four different materials. Table 1 summarizes the results. The initial and the final voltages are the voltage level applied to the samples at the beginning of the test and the voltage level where the first sample failed, respectively. The classification of the material according to [7] corresponds to IEC Class Method used to apply the voltage/end-point criterion/ maximum level of voltage withstood.

Table.1 - Tracking resistance tests: classification of the material according to the test results.

\begin{tabular}{|c|c|c|c|}
\hline Material & $\begin{array}{c}\text { Initial } \\
\text { voltage } \\
{[\mathbf{k V}]}\end{array}$ & $\begin{array}{c}\text { Final } \\
\text { Voltage } \\
{[\mathbf{k V}]}\end{array}$ & IEC Class \\
\hline Santoprene & 3.5 & 4.5 & $2 \mathrm{~A} 4.25 \mathrm{kV}$ \\
\hline Silicone rubber & 3 & 4.5 & $2 \mathrm{~A} 4.25 \mathrm{kV}$ \\
\hline PUR 8070-3 & 3 & 4.75 & $2 \mathrm{~A} 4.5 \mathrm{kV}$ \\
\hline EPDM 2165-1 & 1 & 1 & Failed \\
\hline
\end{tabular}

The PUR material reached the highest voltage level before failure, followed by the Santoprene and silicone rubber, which show similar results. The EPDM material failed at the lowest level of voltage, and it is therefore out of the range of the IEC classification.

The end-point criterion chosen for the tracking tests depends only on the level of current flowing through the sample. Still, it is relevant for this investigation to look at the erosion of the samples after the tests, since it varies considerably in materials that withstand a similar level of voltage. Figs. 7 to 10 show the surface erosion of the samples after the tests.

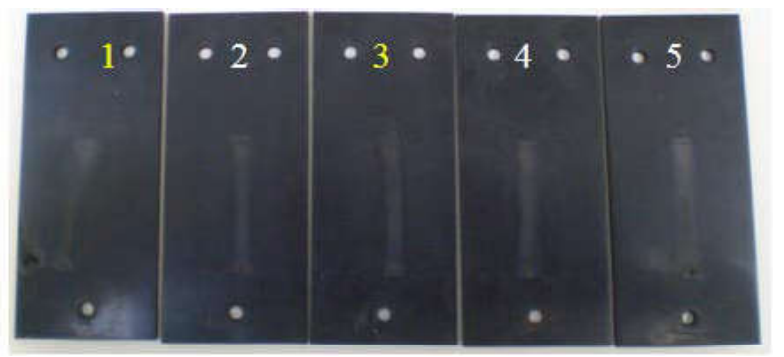

Fig.7 - Surface erosion in Santoprene rubber material after the tracking resistance tests. Samples 1 and 3 failed.

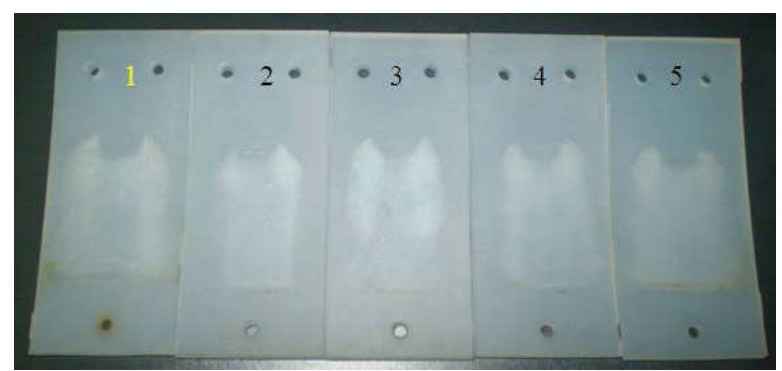

Fig.8 - Surface erosion in Silicone rubber material after the tracking resistance tests. Sample 1 failed. 


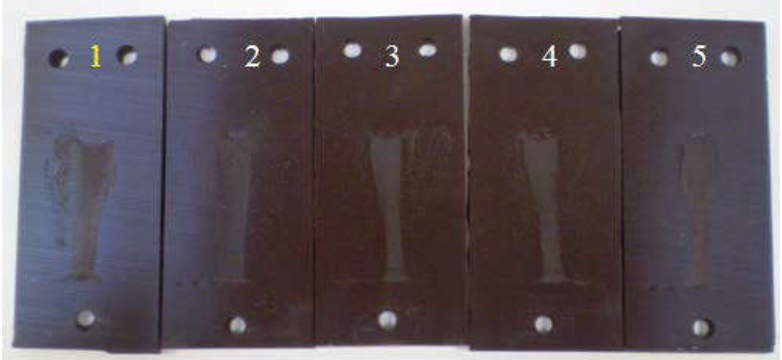

Fig.9 - Surface erosion in PUR rubber material after the tracking resistance tests. Sample 1 failed.

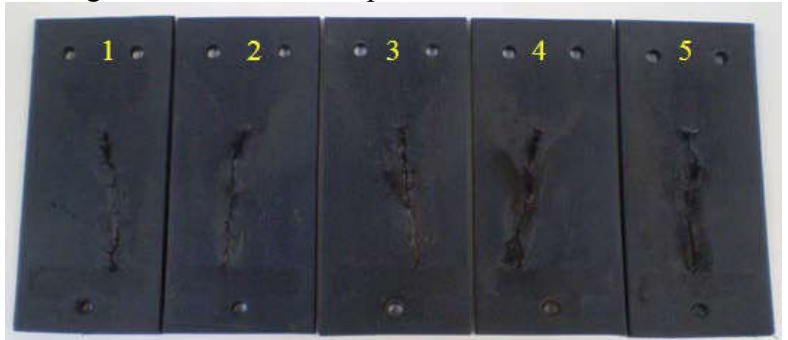

Fig.10 - Surface erosion in EPDM rubber material after the tracking resistance tests. All samples failed.

It is seen in the pictures (Figs. 7-10) that all the samples show a matt appearance between the electrodes. However, this band is different in each material, and only some materials present a deep track. The Santoprene and EPDM materials have a narrow track. The Santoprene samples present erosion only next to the bottom electrode, while the EPDM samples have severe erosion in the whole path between the electrodes. The silicone and PUR materials have a wider dry band and no significant erosion. A failed sample of each material is shown in Fig. 11.

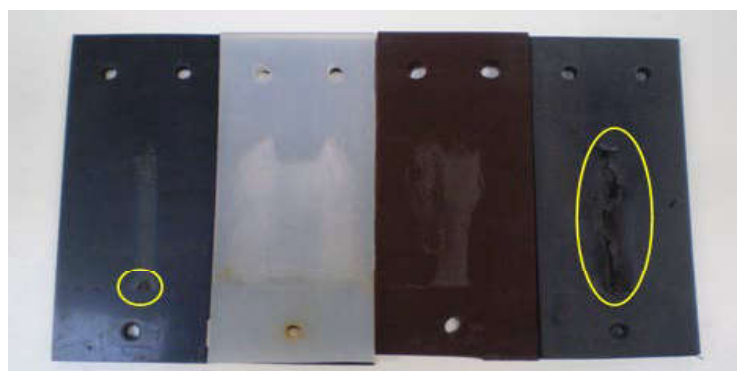

Fig.11 -The surface erosion (marked in yellow) and dry band can be compared in failed samples of each material. From left to right: Santoprene, Silicone rubber, PUR, EPDM.

\section{Discussion}

In this paper, the assessment of materials is done by comparison of the tests results regarding break down and tracking. Though being relevant for wind turbine flap application, the tests themselves do not directly provide information to determine if the material is suitable for the flap application with respect to interaction with lightning discharges. The breakdown strength tests show that the Santoprene material performs better than the other rubber materials, and its performance is comparable to the fiberglass materials used in wind turbine blades [3]. It is also observed that the thickness of the sample has a significant impact on the breakdown strength of the material due to the volume effect. Regarding the tracking resistance tests, all rubber materials reach similar levels of voltage, except the EPDM material, which failed at the beginning of the tests. Furthermore, significant differences in erosion are observed in the materials that withstand the same level of voltage. Finally, it has to be considered that a rubber flap installed in a blade in service will be subjected to mechanical fatigue. Therefore mechanical tests should be done with the tested samples in order to evaluate how the erosion due to tracking affects the performance of the material in general.

\section{Conclusions}

Breakdown and tracking tests were performed on a selection of rubber materials following the same procedure as in GFRP materials for wind turbine blades. The tests results show that the Santoprene material performs better than the other materials, and has comparable properties to the fiberglass material used in blades with respect to tracking resistance and breakdown strength. Therefore, it can be considered as a suitable candidate for wind turbine blade flaps application, regarding its performance in interaction with lightning discharges.

\section{References}

[1] Andersen, P.B. "Advanced load alleviation for wind turbines using adaptative trailing edge flaps: sensoring and control" Risø - DTU, National Laboratory for Sustainable Energy, Technical University of Denmark, $\mathrm{PhD}$ thesis 2010

[2] "IEC 61400-24 Ed.1.0: Wind turbines - Part 24: Lightning protection", IEC, June 2010

[3] Madsen, S.F. "Interaction between electrical discharges and materials for wind turbine blades particularly related to lightning protection" Ørsted - DTU, Electric Power Engineering, Technical University of Denmark, PhD thesis 2006

[4] Candela, A. Holboell, J. Henriksen, M. "Breakdown and tracking properties of rubber materials for wind turbine blades", IEEE International Symposium on Electrical Insulators, June 2012

[5] "IEC 60060-1 Ed.3.0: High voltage test tecniques - Part 1: General definitions and test requirements", IEC, September 2010

[6] "IEC 60243-3 Ed.2.0: Electric strength of insulating materials - Test methods - Part 3: Additional requirements for 1,2/50 impulse tests", July 2001

[7] "IEC 60587 Ed.3.0: Electrical insulating materials used under severe ambient conditions - Test methods for evaluating resistance to tracking and erosion", IEC, May 2007 